\title{
Increased Nucleus Accumbens Volume in First-Episode Psychosis
}

Authors:

Mireia Forns-Nadal ${ }^{a}$, Daniel Bergé ${ }^{* b, c, d}$, Federico Sem ${ }^{e}$, Anna Mané, ${ }^{, b, d}$, Laura Igual ${ }^{e, f}$,Dani Guinart $^{b, c}$, Oscar Vilarroya ${ }^{b, c}$

a. Department of Cognitive Neuroscience and Information Technologies, Internet Interdisciplinary Institute, Open University of Catalonia, Barcelona, Spain

b. Neuroscience group, Hospital del Mar Medical Research Institute (IMIM), Barcelona, Spain

c. Departament de Psiquiatria i Medicina Legal, Facultat de Medicina, Universitat Autònoma de Barcelona, Campus de Bellaterra, Cerdanyola del Vallès, Spain.

d. CIBERSAM, Barcelona, Spain

e. Department of Applied Mathematics and Analysis of the University of Barcelona, Barcelona, Spain

f. Computer Vision Center, Edificio O, Universitat Autònoma de Barcelona, Campus de Bellaterra, s/n, Cerdanyola del Vallès, Spain

* Corresponding author (Daniel Bergé):

Phone: + 3493248 3175, fax: + 34932483445

e-mail: dbergeba@gmail.com

Address: Department of Psychiatry, Hospital del Mar, Passeig Maritim 25-29, 08003, Barcelona, Spain. 


\begin{abstract}
:
Nucleus accumbens has been reported as a key structure in the neurobiology of schizophrenia. Studies analyzing structural abnormalities have shown conflicting results, possibly related to confounding factors. We investigated the nucleus accumbens volume using manual delimitation in first-episode psychosis (FEP) controlling for age, cannabis use and medication. Thirty-one FEP subjects who were naive or minimally exposed to antipsychotics and a control group were MRI scanned and clinically assessed from baseline to 6 months of follow-up. FEP showed increased relative and total accumbens volumes. Clinical correlations with negative symptoms, duration of untreated psychosis and cannabis use were not significant.
\end{abstract}

Keywords: schizophrenia - reward - region of interest - neuroimgaing 


\section{Introduction}

Several studies have suggested the reward system in general, and the nucleus accumbens in particular, as a key role in the neurobiology of schizophrenia. The nucleus accumbens seems to be implicated in the association of stimulus with subsequent reward, generating a process of reinforcement learning (stimulus-reward learning) which is implicated in every-day actions (Berridge and Robinson, 1998). Its dysfunction could explain both aberrant salience attribution, which is thought to underlie delusions (Morrison and Murray, 2009), and an inability to process rewards from everyday life, causing amotivation (Waltz and Gold, 2016), one of the central domains of negative symptoms in schizophrenia.

To date, brain imaging studies investigating the structure and volume of the nucleus accumbens in patients with psychosis have shown controversial results, including increased nucleus accumbens volume in post-mortem schizophrenic subjects (Mamah et al., 2007; Spoletini et al., 2011; Tamagaki et al., 2005; Womer et al., 2014), decreased or no differences in first episode psychosis in relation to control subjects (Ballmaier et al., 2008; Glenthoj et al., 2007; Gunduz et al., 2002). Also, attempts to study possible confounding factors have found increased, decreased or no change with typical or atypical antipsychotics (Boonstra et al., 2011; Ebdrup et al., 2010; Smieskova et al., 2009), and basal ganglia volumes increases or decreases associated to cannabis use (Gilman et al., 2014; Yücel et al., 2008).

The present study was designed to measure manually the volumes of the nuclei accumbens in a sample of early diagnosed FEP compared to a control group, controlling for cannabis use, course of illness and medication. We also aimed to determine the degree of association between accumbens volume and clinical features. We hypothesized that first-episode psychosis patients would show reduced accumbens volumes, compared to those of healthy controls. Additionally, we hypothesized that the reduction in accumbens volumes would be associated with more negative symptoms at baseline and during follow-up.

\section{Methods}

Thirty-one antipyshcotic-naive FEP were recruited between 2008 and 2012 from the emergency service and the inpatient acute ward of Hospital del Mar in Barcelona. Other inclusion criteria were age between 18 and 30 years, absence of severe neurological disorder or head trauma and absence of severe stimulants, opioid or alcohol dependence. Twenty-seven healthy controls without any present axis I disorder or any past severe mental disorder were also recruited. Informed consent was obtained from all subjects prior to entering the study and after receiving approval from the institutional ethical board. This work was carried out in accordance with The Code of Ethics of the Declaration of Helsinki and the Uniform Requirements for manuscripts submitted to biomedical journals.

Patients were clinically assessed using the Positive and Negative Syndrome Scale (PANSS) and Global Assessment of Functioning Scale (GAF) at admission and during the follow-up period (2 and 6 months) by experienced clinical psychiatrists. Psychiatric clinical diagnosis was assessed using the Structured Clinical Interview for DSM-IV (SCID), a minimum of 1 month after admission.

All subjects underwent an MRI scan at study entry. Brain images were acquired using a $1.5 \mathrm{~T}$ Scanner (Phillips). Acquisition parameters of the three-dimensional data set were as follows: high-resolution T1-weighted images with axial acquisition, $\mathrm{TR}=18 \mathrm{~ms}, \mathrm{TE}=4.6 \mathrm{~ms}, \mathrm{TI}=360$, flip angle $=30$, bandwidth $=35, \mathrm{FOV}=22.0$ $\mathrm{cm}$, matrix $=2569256,100$ slices, slice thickness $=1.4, \mathrm{NEX}=1$ and acquisition time $=303100$, resulting voxel size $=0.86 \times 0.86 \times 1.4 \mathrm{~mm}$.

Images were visually inspected to ensure quality and were standardized into $1 \mathrm{x} 1 \mathrm{x} 1$ voxel size. The accumbens was delineated with MriCron (Rorden et al., 2007) by applying previously established criteria (Ballmaier et al., 2008; Carmona et al., 2009; Gunduz et al., 2002). Region of interest delimitation was outlined in all brains by a single investigator (M.F.) blind to group status. Previously, intrarater and interrater reliability (two raters: M.F. and F.S.) was established (intraclass correlation coefficient (ICC) > 0.90) by independent blinded measurements of 10 training scans (Boonstra et al., 2011). Total intracranial volume (TIV) for each subject was calculated using the segmentation algorithms of SPM5 and computing the sum of each segmentation tissue. The relative accumbens volume was computed with the ratio between volume of the ROI and total brain volume (Accumbens volume/TIV). Comparisons between groups were done using t-test for socio-demographic continuous variables and chi-square for categorical variables. Volumes were compared between groups using linear regression correcting for age and weekly cannabis use. 


\section{Results}

Groups were homogeneous with respect to sex (males: $51.6 \%$ in the FEP group vs. $51.9 \%$ in the control group; $\mathrm{p}=$ Non significant $(\mathrm{NS})$ ), civil status (singles: $83.9 \%$ vs. $74.10 \%, \mathrm{p}=\mathrm{NS}$ ), education (basic studies completed: $80.6 \%$ vs. $88.9 \%$, p=NS), handedness (right handed: $83.9 \%$ vs. $81.50 \%, \mathrm{p}=\mathrm{NS}$ ) and average cannabis use ( $2.5 \pm 5.3$ vs. $0.5 \pm 1.5$ units per week, $\mathrm{p}=\mathrm{NS})$; although there were statistical differences in mean age $(26.2 \pm 5.2$ vs. 30.0 \pm 4.2 years old, $\mathrm{p}<0.005$ ). Mean and standard deviation (SD) of duration of untreated psychosis (DUP) was 73.7 \pm 101.4 days, mean and SD score in PANSS negative was 13.4 \pm 5.1 and mean ans SD GAF score was 63.1 \pm 12.9. Mean days and SD from admission to MRI scan were $27.5 \pm 10.4$ days. Patients were treated with antipsychotics during this period (12 subjects were treated with risperidone, 10 with olanzapine, 4 with aripiprazole, 1 with quetiapine and 4 subjects were completely naive to antipsychotics at the moment of the scan). There was no significant correlation between cumulative dose of clorpromazine equivalents and absolute or relative accumbens volume (results not shown).

Compared to healthy controls, FEP had an increased relative (accumbens volume/TIV) and total volume in left and right accumbens (see Table 1). Also FEP had a decreased total intracranial volume (TIV) and grey matter volume. Relative accumbens volume did not significantly correlated to DUP (Pearson coef: -0.169 and -0.198 for right and left accumbens respectively), score of PANSS negative at baseline (Pearson coef: 0.081 and -0.151), at 2 months (Pearson coef:-0.259 and -0.281) and at 6 months (Pearson coef: -0.028 and -0.051) for right and left accumbens respectively.

Table 1: Brain volumes and nucleus accumbens volumes comparisons between first-episode psychosis patients and healthy controls adjusted by age and weekly cannabis use using linear regression.

\begin{tabular}{|l|l|l|l|l|l|}
\hline Brain volumes & $\begin{array}{l}\text { First-episode } \\
\text { psychosis }\end{array}$ & Healthy controls & t-test & df & $\mathrm{p}$ \\
\hline $\begin{array}{l}\text { Total intracranial volume, } \\
\mathrm{ml}(\mathrm{M} / \mathrm{SD})\end{array}$ & $1602.630 / 188.85$ & $1735.411 / 155.45$ & -2.918 & 57 & $\mathrm{p}<0.01$ \\
\hline GM volume, ml (M/SD) & $732.547 / 83.63$ & $775.264 / 71.47$ & -2.690 & 57 & $\mathrm{p}<0.01$ \\
\hline WM volume, ml (M/SD) & $454.661 / 52.04$ & $463.926 / 48.06$ & -0.395 & 57 & $\mathrm{NS}$ \\
\hline Total Accumbens volumes & & & & & \\
\hline Right volume, ml (M/SD) & $0.266 / 0.011$ & $0.244 / 0.01$ & 9.250 & 57 & $\mathrm{p}<0.01$ \\
\hline Left volume, ml (M/SD) & $0.269 / 0.01$ & $0.241 / 0.01$ & 9.981 & 57 & $\mathrm{p}<0.01$ \\
\hline $\begin{array}{l}\text { Relative Accumbens } \\
\text { volumes }\end{array}$ & & & & & \\
\hline $\begin{array}{l}\text { Ratio Right Accumbens } \\
\text { Volume/TIV (M/SD) }\end{array}$ & $0.168 / 0.02$ & $0.141 / 0.01$ & 5.535 & 57 & $\mathrm{p}<0.01$ \\
\hline $\begin{array}{l}\text { Ratio Left Accumbens } \\
\text { Volume/TIV (M/SD) }\end{array}$ & $0.170 / 0.02$ & $0.140 / 0.01$ & 6.093 & 57 & $\mathrm{p}<0.01$ \\
\hline
\end{tabular}

M/SD: Mean, standard deviation; NS: Non-significant p > 0.05; GM: grey matter; WM: white matter

\section{Discussion}




\section{Increased Nucleus Accumbens Volume in First-Episode Psychosis}

First-episode subjects briefly exposed or naive to antipsychotic drugs showed an increased bilateral accumbens volume in comparison to healthy controls once controlling for age, sex and cannabis use. This was a novel result compared to previous studies that found either no differences in volume (Ballmaier et al., 2008; Bois et al., 2015) or a smaller volume in FEP compared to controls although using different methods of measurement. We used manual delimitation of the accumbens with previously validated coordinates and inter-rater validation, whereas studies with the opposite results have used automatic or semi-automatic methods (Gunduz et al., 2002). Although manual tracing and automated measures have shown moderate to strong correlations for medium or extended regions (Arnold et al., 2015), measures of accumbens and small volume regions have shown values of 3 standard deviation discrepancy between automatic methods and the gold standard represented by manual delimitation (Babalola et al., 2009).

In many of the previous studies, most of the patients already fulfill the diagnostic criteria for schizophrenia and not only FEP (schizophreniphorm or brief psychotic disorder) (Bogerts et al., 1985; Mamah et al., 2007; Spoletini et al., 2011; Tamagaki et al., 2005; Womer et al., 2014), making more difficult to discard the confounding factor of the course of illness.

Our patients were naive or minimally treated with atypical antipsychotics, and in agreement to previous reviews, we did not find a correlation between atypical antipsychotic exposure and accumbens volume(Ebdrup et al., 2010; Smieskova et al., 2009), whereas typical antipsychotics may increase basal ganglia medication (Ebdrup et al., 2010; Smieskova et al., 2009). On the contrary, Boonstra et al. (Boonstra et al., 2011) found an increase in the nucleus accumbens of patients who were on atypical antipsychotics compared to those who discontinued, importantly, after an average period of one year on antipsychotic drugs.

Most of the studies excluded substance use disorder in the sample, but they did not control for cannabis use in patients that do not necessarily fulfill abuse or dependence criteria. Cannabis use has been reported to modify brain volume in general (Rapp et al., 2012) and increase accumbens volume in particular (Gilman et al., 2014). Moreover, an increased sensitivity to gray matter change due to the interaction between cannabis use and psychosis has been reported (Malchow et al., 2013). All together suggests that both the acute state in the first episode, antipsychotic treatment and cannabis use could increase or even potentiate one to each other to increase accumbens volume. These changes may be one of the earlier development modifications after the onset of psychosis, as some authors suggested (Haijma et al., 2013; Kahn and Sommer, 2015), and may occur at different time periods and not immediately in the very early first episode, as we have not found accumbens volume association with cannabis use or antipsychotic exposure. This could correspond to an upregulation of the postsynaptic striatal neurons due to hyperdopaminergia in the psychotic state (Abi-Dargham et al., 2000) and cannabis use (Bossong et al., 2009), and post-synpatic dopamine blockade due to antipsychotics (Clow et al., 1980), although this hypothesis outrages the aims of this study. Functional studies using specific task design would help to clarify not only structural but also functional abnormalities of nucleus accumbens in reward processing in schizophrenia.

Conflicts of interest or disclosure form: The author declares no conflict of interest. From other authors, D. Bergé has received honoraria as Speaker and consultory board from Otsuka Pharmaceuticals and Janssen Cilag.

Ethical standards: Study has been approved by the appropriate ethics committee and has therefore been performed in accordance with the ethical standards laid down in the 1964 Declaration of Helsinki and its later amendments. 


\section{References}

Abi-Dargham, A., Rodenhiser, J., Printz, D., Zea-Ponce, Y., Gil, R., Kegeles, L.S., Weiss, R., Cooper, T.B., Mann, J.J., Van Heertum, R.L., Gorman, J.M., Laruelle, M., 2000. Increased baseline occupancy of D2 receptors by dopamine in schizophrenia. Proc. Natl. Acad. Sci. U. S. A. 97, 8104-8109.

Arnold, S.J.M., Ivleva, E.I., Gopal, T.A., Reddy, A.P., Jeon-Slaughter, H., Sacco, C.B., Francis, A.N., Tandon, N., Bidesi, A.S., Witte, B., Poudyal, G., Pearlson, G.D., Sweeney, J.A., Clementz, B.A., Keshavan, M.S., Tamminga, C.A., 2015. Hippocampal volume is reduced in schizophrenia and schizoaffective disorder but not in psychotic bipolar I disorder demonstrated by both manual tracing and automated parcellation (FreeSurfer). Schizophr. Bull. 41, 233-249. doi:10.1093/schbul/sbu009

Babalola, K.O., Patenaude, B., Aljabar, P., Schnabel, J., Kennedy, D., Crum, W., Smith, S., Cootes, T., Jenkinson, M., Rueckert, D., 2009. An evaluation of four automatic methods of segmenting the subcortical structures in the brain. NeuroImage 47, 1435-1447. doi:10.1016/j.neuroimage.2009.05.029

Ballmaier, M., Schlagenhauf, F., Toga, A.W., Gallinat, J., Koslowski, M., Zoli, M., Hojatkashani, C., Narr, K.L., Heinz, A., 2008. Regional patterns and clinical correlates of basal ganglia morphology in non-medicated schizophrenia. Schizophr. Res. 106, 140-147. doi:10.1016/j.schres.2008.08.025

Berridge, K.C., Robinson, T.E., 1998. What is the role of dopamine in reward: hedonic impact, reward learning, or incentive salience? Brain Res. Brain Res. Rev. 28, 309-369.

Bogerts, B., Meertz, E., Schönfeldt-Bausch, R., 1985. Basal ganglia and limbic system pathology in schizophrenia. A morphometric study of brain volume and shrinkage. Arch. Gen. Psychiatry 42, 784-791.

Bois, C., Levita, L., Ripp, I., Owens, D.C.G., Johnstone, E.C., Whalley, H.C., Lawrie, S.M., 2015. Hippocampal, amygdala and nucleus accumbens volume in first-episode schizophrenia patients and individuals at high familial risk: A cross-sectional comparison. Schizophr. Res. 165, 45-51. doi:10.1016/j.schres.2015.03.024

Boonstra, G., van Haren, N.E.M., Schnack, H.G., Cahn, W., Burger, H., Boersma, M., de Kroon, B., Grobbee, D.E., Hulshoff Pol, H.E., Kahn, R.S., 2011. Brain volume changes after withdrawal of atypical antipsychotics in patients with first-episode schizophrenia. J. Clin. Psychopharmacol. 31, 146-153. doi:10.1097/JCP.0b013e31820e3f58

Bossong, M.G., van Berckel, B.N.M., Boellaard, R., Zuurman, L., Schuit, R.C., Windhorst, A.D., van Gerven, J.M.A., Ramsey, N.F., Lammertsma, A.A., Kahn, R.S., 2009. Delta 9-tetrahydrocannabinol induces dopamine release in the human striatum. Neuropsychopharmacol. Off. Publ. Am. Coll. Neuropsychopharmacol. 34, 759766. doi:10.1038/npp.2008.138

Carmona, S., Proal, E., Hoekzema, E.A., Gispert, J.-D., Picado, M., Moreno, I., Soliva, J.C., Bielsa, A., Rovira, M., Hilferty, J., Bulbena, A., Casas, M., Tobeña, A., Vilarroya, O., 2009. Ventro-striatal reductions underpin symptoms of hyperactivity and impulsivity in attention-deficit/hyperactivity disorder. Biol. Psychiatry 66, 972977. doi:10.1016/j.biopsych.2009.05.013

Clow, A., Theodorou, A., Jenner, P., Marsden, C.D., 1980. Cerebral dopamine function in rats following withdrawal from one year of continuous neuroleptic administration. Eur. J. Pharmacol. 63, 145-157.

Ebdrup, B.H., Glenthøj, B., Rasmussen, H., Aggernaes, B., Langkilde, A.R., Paulson, O.B., Lublin, H., Skimminge, A., Baaré, W., 2010. Hippocampal and caudate volume reductions in antipsychotic-naive firstepisode schizophrenia. J. Psychiatry Neurosci. JPN 35, 95-104.

Gilman, J.M., Kuster, J.K., Lee, S., Lee, M.J., Kim, B.W., Makris, N., van der Kouwe, A., Blood, A.J., Breiter, H.C., 2014. Cannabis use is quantitatively associated with nucleus accumbens and amygdala abnormalities in young adult recreational users. J. Neurosci. Off. J. Soc. Neurosci. 34, 5529-5538. doi:10.1523/JNEUROSCI.4745-13.2014

Glenthoj, A., Glenthoj, B.Y., Mackeprang, T., Pagsberg, A.K., Hemmingsen, R.P., Jernigan, T.L., Baaré, W.F.C., 2007. Basal ganglia volumes in drug-naive first-episode schizophrenia patients before and after short- 
term treatment with either a typical or an atypical antipsychotic drug. Psychiatry Res. 154, 199-208. doi:10.1016/j.pscychresns.2006.10.002

Gunduz, H., Wu, H., Ashtari, M., Bogerts, B., Crandall, D., Robinson, D.G., Alvir, J., Lieberman, J., Kane, J., Bilder, R., 2002. Basal ganglia volumes in first-episode schizophrenia and healthy comparison subjects. Biol. Psychiatry 51, 801-808.

Haijma, S.V., Van Haren, N., Cahn, W., Koolschijn, P.C.M.P., Hulshoff Pol, H.E., Kahn, R.S., 2013. Brain volumes in schizophrenia: a meta-analysis in over 18000 subjects. Schizophr. Bull. 39, 1129-1138. doi:10.1093/schbul/sbs118

Kahn, R.S., Sommer, I.E., 2015. The neurobiology and treatment of first-episode schizophrenia. Mol. Psychiatry 20, 84-97. doi:10.1038/mp.2014.66

Malchow, B., Hasan, A., Schneider-Axmann, T., Jatzko, A., Gruber, O., Schmitt, A., Falkai, P., Wobrock, T., 2013. Effects of cannabis and familial loading on subcortical brain volumes in first-episode schizophrenia. Eur. Arch. Psychiatry Clin. Neurosci. 263 Suppl 2, S155-168. doi:10.1007/s00406-013-0451-y

Mamah, D., Wang, L., Barch, D., de Erausquin, G.A., Gado, M., Csernansky, J.G., 2007. Structural analysis of the basal ganglia in schizophrenia. Schizophr. Res. 89, 59-71. doi:10.1016/j.schres.2006.08.031

Morrison, P.D., Murray, R.M., 2009. From real-world events to psychosis: the emerging neuropharmacology of delusions. Schizophr. Bull. 35, 668-674. doi:10.1093/schbul/sbp049

Rapp, C., Bugra, H., Riecher-Rössler, A., Tamagni, C., Borgwardt, S., 2012. Effects of cannabis use on human brain structure in psychosis: a systematic review combining in vivo structural neuroimaging and post mortem studies. Curr. Pharm. Des. 18, 5070-5080.

Rorden, C., Karnath, H.-O., Bonilha, L., 2007. Improving lesion-symptom mapping. J. Cogn. Neurosci. 19, 1081-1088. doi:10.1162/jocn.2007.19.7.1081

Smieskova, R., Fusar-Poli, P., Allen, P., Bendfeldt, K., Stieglitz, R.D., Drewe, J., Radue, E.W., McGuire, P.K., Riecher-Rössler, A., Borgwardt, S.J., 2009. The effects of antipsychotics on the brain: what have we learnt from structural imaging of schizophrenia?--a systematic review. Curr. Pharm. Des. 15, 2535-2549.

Spoletini, I., Cherubini, A., Banfi, G., Rubino, I.A., Peran, P., Caltagirone, C., Spalletta, G., 2011. Hippocampi, thalami, and accumbens microstructural damage in schizophrenia: a volumetry, diffusivity, and neuropsychological study. Schizophr. Bull. 37, 118-130. doi:10.1093/schbul/sbp058

Tamagaki, C., Sedvall, G.C., Jönsson, E.G., Okugawa, G., Hall, H., Pauli, S., Agartz, I., 2005. Altered white matter/gray matter proportions in the striatum of patients with schizophrenia: a volumetric MRI study. Am. J. Psychiatry 162, 2315-2321. doi:10.1176/appi.ajp.162.12.2315

Waltz, J.A., Gold, J.M., 2016. Motivational Deficits in Schizophrenia and the Representation of Expected Value. Curr. Top. Behav. Neurosci. 27, 375-410.doi:10.1007/7854_2015_385

Womer, F.Y., Wang, L., Alpert, K.I., Smith, M.J., Csernansky, J.G., Barch, D.M., Mamah, D., 2014. Basal ganglia and thalamic morphology in schizophrenia and bipolar disorder. Psychiatry Res. 223, 75-83. doi:10.1016/j.pscychresns.2014.05.017

Yücel, M., Solowij, N., Respondek, C., Whittle, S., Fornito, A., Pantelis, C., Lubman, D.I., 2008. Regional brain abnormalities associated with long-term heavy cannabis use. Arch. Gen. Psychiatry 65, 694-701.

doi:10.1001/archpsyc.65.6.694 